\title{
PREVALENCE OF LINGUATULA SERRATA NYMPHS IN SLAUGHTERED SHEEP FROM TEHRAN PROVINCE, IRAN
}

\author{
S. BOKAIE ${ }^{1}$, A. KHANJARI ${ }^{1}$, M.-H. RABIEE ${ }^{1}$, B. HAJIMOHAMMADI ${ }^{2}$, \\ S. SHIRALI ${ }^{3} \&$ G. NEMATI ${ }^{1}$
}

${ }^{1}$ Department of Food Hygiene, Faculty of Veterinary Medicine, University of Tehran, Tehran, Iran; ${ }^{2}$ Department of Food Hygiene and Safety, Faculty of Health, Shahid Sadoughi University of Medical Sciences, Yazd, Iran; ${ }^{3}$ Department of Parasitology, Faculty of Veterinary Medicine, Islamic Azad University, Science \& Research Branch, Tehran, Iran.

\section{Summary}

Bokaie, S., A. Khanjari, M.-H. Rabiee, B. Hajimohammadi, S. Shirali \& G. Nemati, 2019. Prevalence of Linguatula serrata nymphs in slaughtered sheep from Tehran province, Iran. Bulg. J. Vet. Med., 22, No 4, 487-492.

Linguatulosis is a zoonotic disease caused by Linguatula serrata. Ruminants are the most important intermediate hosts of this parasite. This study was carried out to evaluate the prevalence rate of Linguatula serrata infection in slaughtered sheep in slaughterhouses of Tehran province, Iran. A total of 767 slaughtered sheep were selected in 2016 using systematic random sampling and their mesenteric lymph nodes were examined for Linguatula serrata nymph detection. Then, the infection rate and its intensity in different ages, sex and seasons were assessed. It was revealed that 66 sheep $(8.6 \%)$ were infected with Linguatula serrata nymphs. Intensity of the infection was 4.88 nymphs. The highest rate of infection was observed in sheep aged over 19 months $(\mathrm{P} \leq 0.05)$. The highest infection rate and intensity of the infection were recorded in winter and summer, respectively $(P \leq 0.05)$. Besides, intensity of the infection in females was significantly higher than that in males $(\mathrm{P} \leq 0.05)$. This study indicates the importance of Linguatula serrata infection in sheep in Tehran province, and the need to improve preventive measures for reducing the rate of infection and preventing transmission of the infection from sheep and other ruminant to other animals and humans in Iran.

Key words: linguatulosis, L. serrata, sheep

\section{INTRODUCTION}

Linguatulosis is considered as a parasitic and zoonotic disease. The most commonly reported species involved in linguatulosis is Linguatula serrata which is widespread throughout the globe but in most cases is found in the Middle East (Roberts \& Janovy, 2005; Shamsi et al., 2017). This parasite belongs to the phylum Pentastomida and was first detected in 1789 by Frohlich (Ravindran et al., 2008). The parasite body is broad and flat, looking like a tongue. This white-coloured parasite is wide in the anterior and narrow in the posterior part. The parasite has sepa- 
rate sexes; the female is larger than its male counterpart (Roberts \& Janovy, 2005).

This species is a common parasite of humans and livestock. Adult forms of the parasite live in the nasal passages and respiratory tract of the final hosts such as dogs, foxes, and wolves. Ruminants are the most important intermediate host of this parasite and they are infected by ingesting parasite eggs; after that, the larval stage of the parasite ( $P$. denticulatum) may survive in the gut and internal organs, especially the intestinal (mesenteric) lymph nodes, liver, lungs, spleen and kidneys (Alcala-Canto et al., 2007; Shamsi et al., 2017). Humans serve as either intermediate or final hosts and can be infected by both adult and nymph form of parasite; however, the infection with the former is rare. The two forms of the infection are: visceral linguatulosis, following consumption of water, vegetables or fruits contaminated with the eggs, and nasopharyngeal linguatulosis, known also as Halzoun or Marrara syndrome, following consumption of raw or undercooked infected viscera of infected animals (Shekarforoush et al., 2004; David et al., 2006; Tajik et al., 2006; Koehsler et al., 2011; Pirali Kheirabadi et al., 2014; Yazdani et al., 2014; Hajimohammadi et al., 2017).

Recently, L. serrata has got an increasing public health importance, and new cases of human infection are reported from different parts of Iran. However, there is not sufficient information regarding the infection rate of sheep in Tehran province, while the number of slaughtered ruminants in the province is the highest one in Iran. Therefore, the aim of this study is to investigate the prevalence of Linguatula serrata infection in slaughtered sheep in slaughterhouses of Tehran province, Iran.

\section{MATERIALS AND METHODS Sampling}

To conduct the present study, 767 sheep (685 meat sheep and 82 dairy sheep) out of the slaughtered sheep in slaughterhouses of Tehran province were selected using systematic random sampling in 2016. Then, age of the slaughtered sheep (according to dental formula and animal owner remarks) and sex (physical appearance) were determined and samples of mesenteric lymph nodes (5 lymph nodes per animal) were collected. Afterwards, the samples kept in ice were transferred to the lab in less than 1 hour.

\section{Preparation methods}

In the laboratory, adipose tissues surrounding the lymph nodes were removed and the lymph nodes were dissected into smaller pieces by scalpel, making multiple longitudinal cuttings. The resulting small pieces were macroscopically examined for nymphs. For further investigation of the samples, a tissue digestion method was used. In this method, $5 \mathrm{~g}$ of pepsin enzyme was added to $25 \mathrm{~mL}$ hydrochloric acid and diluted with water to a total volume of $1 \mathrm{~L}$. Then, $100 \mathrm{~g}$ of minced tissue was immersed in digestion solution and incubated at $37{ }^{\circ} \mathrm{C}$ for $24 \mathrm{~h}$. After this process, the suspensions were examined under a stereoscope (Shekarforoush et al., 2004).

\section{Statistical analysis}

Results were reported as infection rate and intensity of infection according to different age, sex and season groups. Infection rate in different groups were tested by Chi-square test. Intensity of infection was tested using ANOVA and LSD test. Pvalues less than 0.05 were considered statistically significant. Statistical analyses 
were performed by using SPSS version 19.0 .

\section{RESULTS}

Results of this study showed that 66 out of 767 sheep $(8.6 \%)$ were infected with $\mathrm{Lin}$ guatula serrata nymphs in their mesenteric lymph nodes. Also, intensity of the infection (the average number of Linguatula serrata nymphs in the infected sheep) was 4.88 nymphs.

The infection rates according to different age, season and sex are summarised in Table 1.There was a significant difference concerning the infection rate in three age groups $(\mathrm{P}=0.0001)$. That is, the infection rate in the group older than 19 months of age was significantly higher than those $<12$ months of age $(\mathrm{P}=0.0001)$. The infection rate in the group older than 19 months was significantly higher than those in group of 13-18 months $(\mathrm{P}=0.001)$. Also, significant difference was found regarding the infection rate in different seasons $(\mathrm{P}=0.0001)$. The infection rate in winter was significantly highest in comparison with spring $(\mathrm{P}=0.0001)$, summer $(\mathrm{P}=0.0001)$ and fall $(\mathrm{P}=0.0001)$ while the infection rate in different sexes was not statistically significant $(\mathrm{P}=0.42)$.

Intensity of the infection according to different age, season and sex are summarised in Table 2. Intensity of the infection in different age groups was not statistically significant $(\mathrm{P}=0.10)$. There was a significant difference concerning the intensity of the infection in different seasons $(\mathrm{P}=0.02)$. In summer, the intensity of the infection was significantly higher than that in spring $(\mathrm{P}=0.004)$, fall $(\mathrm{P}=0.009)$ and winter $(\mathrm{P}=0.005)$. Also, intensity of the infection in females was statistically higher than that in males $(\mathrm{P}=0.01)$.

Table 1. Linguatula serrata infection rate in sheep according to age and sex

\begin{tabular}{lccccc}
\hline & \multicolumn{3}{c}{ Age (month) } & \multicolumn{3}{c}{ Sex } \\
\cline { 2 - 6 } & $0-12$ & $12-18$ & $\geq 19$ & Male & Female \\
\hline $\begin{array}{l}\text { No. of sheep } \\
\begin{array}{l}\text { No. (\%) of infected } \\
\text { sheep }\end{array}\end{array}$ & $10(5)$ & $36(7.8)$ & $20(18.5)$ & $58(8.3)$ & $8(11.1)$ \\
$\begin{array}{l}\text { Mean of nymphs in } \\
\text { MLN of infected sheep }\end{array}$ & $3.00 \pm 0.66$ & $3.54 \pm 0.51$ & $8.00 \pm 2.97$ & $3.98 \pm 0.59$ & $11.38 \pm 7.10$ \\
$\begin{array}{l}\text { Median of nymphs in } \\
\text { MLN of infected sheep }\end{array}$ & 3 & & & & \\
\hline
\end{tabular}

Table 2. Linguatula serrata infection rate in sheep according to the season

\begin{tabular}{|c|c|c|c|c|}
\hline & \multicolumn{4}{|c|}{ Season } \\
\hline & Spring & Summer & Fall & Winter \\
\hline No. of sheep & 199 & 187 & 195 & 186 \\
\hline No. $(\%)$ of infected sheep & $11(5.5)$ & $5(2.7)$ & $12(6.2)$ & $38(20)$ \\
\hline $\begin{array}{l}\text { Mean of nymphs in MLN of } \\
\text { infected sheep }\end{array}$ & $2.92 \pm 0.64$ & $15.20 \pm 11.48$ & $3.91 \pm 0.91$ & $4.42 \pm 0.84$ \\
\hline $\begin{array}{l}\text { Median of nymphs in MLN } \\
\text { of infected sheep }\end{array}$ & 2 & 5 & 3 & 3 \\
\hline
\end{tabular}




\section{DISCUSSION}

Since Tehran has several big slaughterhouses and the number of slaughtered ruminants is the highest in Iran, this study on infection rate of slaughtered sheep with Linguatula serrata was carried out in this province. It should be noted that in this research, mesenteric lymph nodes of the sheep were examined because it is the first site that the parasite chooses to enter (Shekarforush \& Arzani-Shahni, 2002). However, based on other conducted studies in Iran, the reported infection rates of sheep mesenteric lymph nodes in various parts of Iran were as followed: Isfahan, 11\% (Kheirabadi et al., 2015); Urmia, 65.7\% (Yakhchali et al., 2009); Shiraz, $11.5 \%$ (Shekarforoush et al., 2004); Tabriz, 52.5\% (Tavassoli et al., 2007) Kermanshah, 19.7\% (Hashemnia et al., 2016). In this research, the infection rate of sheep in their mesenteric lymph nodes was $8.6 \%$. It can be concluded that the infection rate in slaughtered sheep in different parts of Iran varied between $8.6 \%$ and $65.7 \%$ so the infection rate in Tehran province was approximately lower than that in other provinces of the country. Therefore, it is crucial to improve preventive measures to reduce the rate of infection in ruminants as well as to prevent transmission of the infection from ruminants to other animals and humans. For instance, more attention is required when inspecting the internal organs of slaughtered ruminants especially mesenteric lymph nodes in the slaughterhouses of Iran in connection with the several reports for human linguatulosis (Koehsler et al., 2011; Hamid et al., 2012; Yazdani et al., 2014).

In the present study, intensity of the infection (average number of Linguatula serrata nymphs in infected sheep) in slaughtered sheep was 4.88 nymphs. In general, the studies concerning Linguatula serrata infection on sheep in Iran have rarely calculated intensity of the infection.

A plethora of studies carried out either in Iran or other parts of the world confirmed the impact of aging on increased rate of infection with Linguatula serrata parasite in sheep and other livestock (Fard et al., 2012; Kheirabadi et al., 2015; Nematollahi et al., 2015; Hashemnia et al., 2016). In this study, rate of the infection in sheep over 19 months of age was higher than that in younger ones. Considering these findings, the lower level of the infection in young livestock can be dependent on the type of feeding (feeding with milk at an early age instead of grass), the development period of the nymph's of about 5-6 months, and also less frequent contacts of young animals with dogs.

There are contradictions in terms of the impact of the season on the infection rate in different studies. Indeed, some studies didn't identify a significant association between season and Linguatula infection while some other studies found a significant association. For instance, the highest rate of the infection in some studies was in spring (Kheirabadi et al., 2015), while fall had the highest rate of infection in others (Fard et al., 2012). In this study, the infection rate of sheep in winter was significantly higher than that in other seasons. It seems that infection rate of Linguatula serrata in different seasons is related to the climatic conditions of the region. Also, our results revealed that intensity of the infection in summer was significantly higher than that in other seasons.

In this study, significant difference was not found between rate of the infection and sex of sheep. Many studies in sheep also confirm this finding. However, results of some studies indicate that rate of the infection in females is higher than that 
in males (Shekarforoush et al., 2004). The aforementioned difference is attributed to the aging of female animals compared to their opposite-sex counterparts included in the study. Likewise, our results revealed that intensity of the infection in females was significantly higher than in males.

In general, this study indicated the importance of Linguatula serrata infection in sheep in Tehran province, Iran and suggests the need to improve preventive measures for reduction of the rate of infection and prevent the transmission of the infection from sheep and other ruminants to other animals and humans in Iran.

\section{ACKNOWLEDGEMENTS}

The authors acknowledge the Vice Chancellor for Research and Technology of Veterinary College of University of Tehran for funding this research.

\section{REFERENCES}

Alcala-Canto, Y., A. Alberti-Navarro \& F. IbbaraVelarde, 2007. Serin protease activity demonstrated in larval stage of the pentastomid Linguatula serrata. Parasitology Research, 100, 1011-1014.

David, T. J., A. P. William \& J. Petri, 2006. Markell and Voge's Medical Parasitology. Saunders Elsevier, St. Louis, Missouri.

Fard, S. R. N., N. Ghalekhani, R. Kheirandish, S. Fathi \& E. N. Asl, 2012. The prevalence of Linguatula serrata nymphs in camels slaughtered in Mashhad slaughterhouse, Northeast Iran. Asian Pacific Journal of Tropical Biomedicine, 2, 885-888.

Hajimohammadi, B., G. Eslami, S. KhalatbariLimaki, M. H. Ehrampoush, A. Oryan, H. Zandi, \& H. R. Dehghan, 2017. The role of Linguatula serrata nymph in transmission of enteric bacterial pathogens to internal organs in sheep. Journal of Parasitic Diseases, 41, 754-760.
Hamid, T., Y. D. Hossein, B. B. Mehran, F. S. Masood \& E. Hamid. 2012. A case report of Linguatula serrata infestation from rural area of Isfahan city, Iran. Advanced Biomedical Research, 1, 1-3.

Hashemnia, M., F. Rezaei, M. Sayadpour \&Y. Shahbazi, 2016. Prevalence of Linguatula serrata nymphs and pathological lesions of infected mesentric lymph nodes among ruminants in Kermanshah, Western Iran. Bulgarian Journal of Veterinary Medicine, 21, 94-102.

Kheirabadi, K. P., A. A. Fallah, H. Azizi, A. D. Samani \& S. D. Dehkordi, 2015. Prevalence of Linguatula serrata nymphs in slaughtered sheeps in Isfahan province, southwest of Iran. Journal of Parasitic Diseases, 39, 518-521.

Koehsler, M., J. Walochnik, M. Georgopoulos, C. Pruente, W. Boeckeler, H. Auer \&T. Barisani-Asenbauer, 2011. Linguatula serrata tongue worm in human eye, Austria. Emerging Infectious Diseases, 17, 870-872.

Nematollahi, A., H. Rezai, J. Helan \& N. Moghaddam, 2015. Occurrence of Linguatula serrata nymphs in cattle slaughtered in Tabriz, Iran. Journal of Parasitic Diseases, 39, 140-143.

Pirali Kheirabadi, Kh., A. Fallah \& A. Abolghasemi, 2014. Prevalence of Linguatula serrata nymphs in slaughtered goats in Isfahan province. Journal of Veterinary Reseasrch, 8, 79-83.

Ravindran, R., B. Lakshmanan, C. Ravishankar \& H. Subramanian, 2008. Prevalence of Linguatula serrata in domestic ruminants in South India. Southeast Asian Journal of Tropical Medicine Public Health, 39, 808-812.

Roberts, L. S. \& J. R. Janovy, 2005. Foundations of Parasitology, $7^{\text {th }}$ edn, McGrawHill, USA.

Shamsi, S., K. McSpadden, S. Baker, \& D. J. Jenkins, 2017. Occurrence of tongue worm, Linguatula cf. serrata (Pentastomida: Linguatulidae) in wild canids and livestock in south-eastern Australia. Inter- 
Prevalence of Linguatula serrata nymphs in slaughtered sheep from Tehran province, Iran

national Journal for Parasitology: Parasites and Wildlife, 6, 271-277.

Shekarforoush, S., S. Razavi \& M. Izadi, 2004. Prevalence of Linguatula serrata nymphs in sheep in Shiraz, Iran. Small Ruminant Research, 52, 99-101.

Shekarforush, S. \& P. Arzani-Shahni, 2002. Prevalence of Linguatula serrata nymphs in livers of ruminants in Shahrekord slaughterhouse, Iran. Iranian Journal of Veterinary Research, 1, 57-62.

Tajik, H., M. Tavassoli, B. Dalir-Naghadeh \& M. Danehloipour, 2006. Mesenteric lymph nodes infection with Linguatula serrata nymphs in cattle. Iranian Journal of Veterinary Research, 7, 82-85.

Tavassoli, M., H. Tajic, B. Dalir-Naghadeh \& F. Hariri, 2007. Prevalence of Linguatula serrata nymphs and gross changes of infected mesenteric lymph nodes in sheep in Urmia, Iran. Small Ruminant Research, 72, 73-76.

Yakhchali, M., S. H. Athari, B. Hajimohammadi \& M. Raeisi, 2009. Prevalence of Linguatula serrata in the ruminants slaughtered in Urmia slaughterhouse, Iran.
Journal of Veterinary Research, 64, 329332.

Yazdani, R., I. Sharifi, M. Bamorovat \& M. A. Mohammadi, 2014. Human linguatulosis caused by Linguatula serrata in the city of Kerman, South-eastern Iran- Case Report. Iranian Journal of Parasitology, 9, 282 285.

Paper received 07.01.2018; accepted for publication 16.03.2018

\section{Correspondence:}

Saied Bokaie

Department of Food Hygiene,

Faculty of Veterinary Medicine,

University of Tehran, Tehran, Iran

e-mail: sbokaie@ut.ac.ir 